\title{
The Multi-Species Farley-Buneman Instability in the Solar Chromosphere
}

\author{
Chad A. Madsen \\ cmadsen@bu.edu \\ Yakov S. Dimant \\ Meers M. Oppenheim \\ Center for Space Physics, Boston University, 725 Commonwealth Ave., Boston, MA 02215 \\ and \\ Juan M. Fontenla \\ Laboratory for Atmospheric and Space Physics, University of Colorado at Boulder, 1234 \\ Innovation Dr., Boulder, CO 80303
}

Received — 


\begin{abstract}
Empirical models of the solar chromosphere show intense electron heating immediately above its temperature minimum. Mechanisms such as resistive dissipation and shock waves appear insufficient to account for the persistence and uniformity of this heating as inferred from both UV lines and continuum measurements. This paper further develops the theory of the Farley-Buneman Instability (FBI) which could contribute substantially to this heating. It expands upon the single ion theory presented by Fontenla (2005) by developing a multiple ion species approach that better models the diverse, metal-dominated ion plasma of the solar chromosphere. This analysis generates a linear dispersion relationship that predicts the critical electron drift velocity needed to trigger the instability. Using careful estimates of collision frequencies and a one-dimensional, semi-empirical model of the chromosphere, this new theory predicts that the instability may be triggered by velocities as low as $4 \mathrm{~km} \mathrm{~s}^{-1}$, well below the neutral acoustic speed. In the Earth's ionosphere, the FBI occurs frequently in situations where the instability trigger speed significantly exceeds the neutral acoustic speed. From this, we expect neutral flows rising from the photosphere to have enough energy to easily create electric fields and electron Hall drifts with sufficient amplitude to make the FBI common in the chromosphere. If so, this process will provide a mechanism to convert neutral flow and turbulence energy into electron thermal energy in the quiet Sun.
\end{abstract}

Subject headings: Sun: chromosphere - plasmas - instabilities 


\section{Introduction}

Just above the temperature minimum in the solar chromosphere, the plasma temperature rises steeply by over $2000 \mathrm{~K}$. This region of intense heating explained the continuum and line emissions in the quiet-Sun chromosphere, but the energy source remains unexplained (Athay 1966). Detailed semi-empirical models of this region by Vernazza et al. (1981) and Fontenla et al. (1991; 1993; 2009) also predict the enhanced temperature region shown in Figure 1, but did not explain the mechanisms sustaining it.

Researchers have proposed a number of mechanisms to explain the heating but none have proven compelling. Carlsson \& Stein (1992) suggested that acoustic shocks were responsible; however, the predicted temporary variations in the Ca II K line profile remain unobserved (Carlsson 2007). Campos \& Mendes (1995) proposed resistive heating due to steady electric currents or MHD wave dissipation as possible sources of the enhanced temperature region; however, chromospheric plasma does not appear to have a sufficiently high conductivity to produce this region via classical Joule heating (Socas-Navarro 2007).

Fontenla (2005) and Fontenla et al. (2008) suggested that plasma turbulence due to the Farley-Buneman Instability (FBI) can heat some layers of the chromosphere. They argue that convective motions from the photosphere will drag ions across the solar magnetic field and drive the FBI with enough energy to account for upper chromospheric heating. This would explain the radiative losses in both the quiet-Sun internetwork and network lanes. These papers applied an oversimplified approach to the FBI appropriate for the ionosphere but needs some modification to accurately model the instability in the metal-ion dominated chromosphere. The current paper derives a chromospheric FBI which modifies the results of Fontenla (2005) and Fontenla et al. (2008), lending further support for their conclusions.

The FBI was first used to explain density irregularities in the equatorial electrojet observed in ionospheric radar experiments (Farley 1963; Buneman 1963). The instability 
occurs in weakly ionized, collisional plasmas with strongly magnetized electrons but collisionally demagnetized ions. Electrostatic waves develop due to the disparate motions of the electrons and ions when plasma flows across magnetic field lines. The zeroth order motion of the electrons is the $\boldsymbol{E} \times \boldsymbol{B}$ (Hall) drift while the ions necessarily follow the neutral flow because of the high ion-neutral collision rates. Linear wave growth develops if the electron $\boldsymbol{E} \times \boldsymbol{B}$ drift velocity exceeds the ion-acoustic velocity multiplied by a dimensionless factor close to unity. The instability in the ionosphere develops on a time scale somewhat larger than the ion-neutral mean free time, which means it operates on a millisecond to 10s of millisecond time scale. This exceeds Alfvénic time scales but is below most electron frequencies.

The electric field that creates the $\boldsymbol{E} \times \boldsymbol{B}$ drift that drives the FBI in the ionosphere arises from different causes in various regions of the Earth's E-region ionosphere. Near the magnetic equator, the predominant energy source for this derives from strong neutral winds that flow East-West across the largely horizontal North-South pointing geomagnetic field (Richmond 1973). Since the ions must follow the neutrals but the electrons remain mostly tied to field lines, a complex current develops, and that, combined with the vertical gradient in the neutral density and hence the conductivity, causes the formation of strong East-West currents called electrojets. In the Earth's auroral regions, where radars detect ferociously strong FBI waves, electric fields generated in the coupled magnetosphere-ionosphere system propagate down the mostly vertical magnetic fields (Dimant \& Oppenheim 2010a; 2010b). These fields drive auroral electrojets with hypersonic electron flow rates and the FBI then heats the electrons in this region dramatically (Foster \& Erickson 2000; Oppenheim \& Dimant 2013).

This paper expands the theory of the FBI employed by Fontenla (2005), Fontenla et al. (2008), and Gogoberidze et al. (2009) to account for the diversity of ion species in the solar 
chromosphere. The single species theory is appropriate for the E-region ionosphere where the primary ion constituents are $\mathrm{O}_{2}^{+}$and $\mathrm{NO}^{+}$which are similar in mass and can be treated as a single species. However, ionic components of chromospheric plasma range from protons to ionized metals dominated by Si II, Mg II, and Fe II. Assuming an average ion mass ignores the physics arising from differences in mobilities and collision frequencies among the various ion species. This paper derives the linear, multi-species dispersion relation and electron $\boldsymbol{E} \times \boldsymbol{B}$ drift trigger velocity appropriate for chromospheric conditions. It then applies the multi-species theory to a recent model of the solar chromosphere to determine the likely range of electron drift velocities necessary to trigger the instability as a function of pressure within the chromosphere.

\section{Theory}

\subsection{Linear Dispersion Relationship and Trigger Velocity}

A model of the FBI applicable to the chromospheric plasma requires deriving a multi-species dispersion relationship using a linear, fluid approximation similar to that used by Farley (1963) and Buneman (1963), and later refined by Sudan et al. (1973). To obtain the simplest manageable dispersion relationship, we will make several assumptions. First, we assume all ion species are demagnetized and electrons are magnetized. A species is magnetized when its gyrofrequency greatly exceeds its collision frequency. Given the temporal and spatial scales under consideration, we approximate the electric field with an electrostatic field represented by the gradient of a scalar potential. Next, we take all ions to be singly ionized and assume the plasma is quasineutral since we are concerned with

frequencies much smaller than the plasma frequency. We consider electron inertia negligible due the large difference in mass between electrons and any ion species. Also, we consider the ion Pedersen drift negligible allowing the ions to be at rest in the frame of the neutral 
flow to zeroth order. Furthermore, we take the zeroth order electron velocity to be its $\boldsymbol{E} \times \boldsymbol{B}$ drift velocity

Assuming linear, plane wave perturbations, wave propagation will occur predominantly in the direction perpendicular to $\boldsymbol{B}$; in other words, $k_{\|}^{2} \ll k_{\perp}^{2}$ where $\boldsymbol{k}_{\|}$and $\boldsymbol{k}_{\perp}$ are the wavevector components parallel and perpendicular to $\boldsymbol{B}$, respectively. Only long wavelength waves are considered; in other words, $k v_{D} \ll \nu_{j}$ where $v_{D}$ is the electron $\boldsymbol{E} \times \boldsymbol{B}$ drift velocity and $\nu_{j}$ is the total ion momentum transfer collision frequency for the $\mathrm{j}^{\text {th }}$ ion species (Dimant \& Oppenheim 2004). However, these wavelengths are much longer than those characteristic of Alfvénic modes. We assume the plasma has no zeroth order density gradients which could greatly enhance the instability but will lead to a far more complex analysis.

We start our analysis with the fluid equations of motion for both electrons and ions:

$$
\begin{gathered}
n \equiv n_{e}=\sum_{j} n_{j} \\
\frac{\partial n}{\partial t}+\boldsymbol{\nabla} \cdot\left(n \boldsymbol{v}_{\boldsymbol{e}}\right)=0 \\
\frac{\partial n_{j}}{\partial t}+\boldsymbol{\nabla} \cdot\left(n_{j} \boldsymbol{v}_{j}\right)=0 \\
e\left(\boldsymbol{\nabla} \phi-\boldsymbol{v}_{e} \times \boldsymbol{B}\right)-\frac{\gamma_{e} K_{B} T_{e} \boldsymbol{\nabla} n}{n}-m_{e} \nu_{e} \boldsymbol{v}_{e}=0 \\
m_{j}\left[\frac{\partial \boldsymbol{v}_{j}}{\partial t}+\left(\boldsymbol{v}_{\boldsymbol{j}} \cdot \boldsymbol{\nabla}\right) \boldsymbol{v}_{\boldsymbol{j}}\right]=-e \boldsymbol{\nabla} \phi-\frac{\gamma_{j} K_{B} T_{j} \boldsymbol{\nabla} n_{j}}{n_{j}}-m_{j} \nu_{j} \boldsymbol{v}_{j}
\end{gathered}
$$

where $n$ is number density, $\boldsymbol{v}$ is flow velocity, $e$ is the elementary charge, $\phi$ is electrostatic potential, $\boldsymbol{B}$ is the magnetic field, $\gamma$ is the ratio of heat capacities, $K_{B}$ is the Boltzmann constant, $T$ is temperature, $m$ is mass, and the subscript $e$ corresponds to electrons while the subscript $j$ corresponds to the $\mathrm{j}^{\text {th }}$ ion species. We maintain separate temperatures for each species even though collisions will keep the ion temperatures quite similar to the neutral ones. The electron temperature, however, can become somewhat elevated by a 
range of processes. The collision rates, $\nu_{e}$ and $\nu_{j}$, include both collisions with neutrals and coulomb collisions. A full treatment of Coulomb collisions would add a few additional components to eqs (41) and (5), but these only modify our final results by a negligible amount (Gogoberidze et al. 2009). This restricts our analysis to near the temperature minimum where collisions with neutrals dominate over Coulomb collisions.

To find the dispersion relationship, we keep only the first-order, linear terms in eqs (11)-(5). We assume plane wave perturbations such that all dynamical quantities vary as $\xi=\delta \xi \exp [-i(\omega t-\boldsymbol{k} \cdot \boldsymbol{x})]$, providing:

$$
\begin{gathered}
\left(\omega-\boldsymbol{k} \cdot \boldsymbol{v}_{D}\right) \delta n-n_{0}\left(\boldsymbol{k} \cdot \boldsymbol{\delta} \boldsymbol{v}_{\boldsymbol{e}}\right)=0 \\
\omega \delta n_{j}-n_{j 0}\left(\boldsymbol{k} \cdot \boldsymbol{\delta} \boldsymbol{v}_{\boldsymbol{j}}\right)=0 \\
e\left(i \boldsymbol{k} \delta \phi-\boldsymbol{\delta} \boldsymbol{v}_{\boldsymbol{e}} \times \boldsymbol{B}\right)-i \frac{\gamma_{e} K_{B} T_{e}}{n_{0}} \boldsymbol{k} \delta n-m_{e} \nu_{e} \boldsymbol{\delta} \boldsymbol{v}_{\boldsymbol{e}}=0 \\
m_{j}\left(\omega+i \nu_{i}\right) \boldsymbol{\delta} \boldsymbol{v}_{\boldsymbol{j}}-e \boldsymbol{k} \delta \phi-\frac{\gamma_{j} K_{B} T_{j}}{n_{j 0}} \boldsymbol{k} \delta n_{j}=0 \\
\delta n=\sum_{j} \delta n_{j}
\end{gathered}
$$

where the subscript 0 represents zeroth order quantities, and $\delta$ represents a linearly perturbed Fourier coefficient. This analysis is in the neutral fluid rest frame where the electrons have a zero-order velocity of $\boldsymbol{v}_{\boldsymbol{D}} \equiv \boldsymbol{E} \times \boldsymbol{B} /|\boldsymbol{B}|$ and the small ion Pedersen drifts are inconsequential.

Eliminating all of the linearly perturbed Fourier coefficients yields the dispersion relationship:

$$
D(\omega, \boldsymbol{k}) \equiv\left[\omega-\boldsymbol{k} \cdot \boldsymbol{v}_{\boldsymbol{D}}+i \frac{\nu_{e} k^{2} U_{e}^{2}}{\Omega_{e}^{2}}\left(1+\frac{\Omega_{e}^{2} k_{\|}^{2}}{\nu_{e}^{2} k^{2}}\right)\right] \sum_{j} \frac{n_{j 0}}{n_{0} \psi_{j}}\left(\omega-i \frac{\omega^{2}}{\nu_{j}}+i \frac{k^{2} U_{j}^{2}}{\nu_{j}}\right)^{-1}+1=0
$$


where $\Omega$ is the gyrofrequency, $U_{j} \equiv \sqrt{\gamma_{j} K_{B} T_{j} / m_{j}}$ is the thermal velocity, and $\psi_{j}$ is defined as:

$$
\psi_{j} \equiv \frac{\nu_{j} \nu_{e}}{\Omega_{j} \Omega_{e}}\left(1+\frac{\Omega_{e}^{2} k_{1}^{2}}{\nu_{e}^{2} k^{2}}\right) .
$$

Assuming only one ion species reduces eq (11) to the single-ion dispersion relation found in Sudan et al. (1973):

$$
\omega\left(1+\psi_{j}\right)-\boldsymbol{k} \cdot \boldsymbol{v}_{D}=i \frac{\psi_{j}}{\nu_{j}}\left[\omega^{2}-\left(\frac{m_{e}}{m_{j}} U_{e}^{2}+U_{j}^{2}\right) k^{2}\right]
$$

Returning to eq (11), let $\omega \equiv \omega_{r}+i \Gamma$, where $\omega_{r}$ represents the oscillation frequency of the waves and $\Gamma$ represents the growth/damping rate of the waves. To recover a phase-velocity relation from (11), we will ignore all small and imaginary terms by taking $\Gamma \rightarrow 0$ and $\omega_{r} / \nu_{j} \rightarrow 0$ for all ion species $j$. This provides:

$$
\omega_{r}=\frac{\boldsymbol{k} \cdot \boldsymbol{v}_{D}}{1+\psi}
$$

where

$$
\psi \equiv\left(\sum_{j} \frac{n_{j 0}}{n_{0} \psi_{j}}\right)^{-1}
$$

which is equivalent to the oscillation frequency from Sudan et al. (1973). Now, we will find an approximate solution for the growth rate by assuming slow growth $\left(|\Gamma| \ll\left|\omega_{r}\right|\right)$ and expanding (11) about $\Gamma=0$ :

$$
\begin{gathered}
D(\omega, \boldsymbol{k})=\operatorname{Re}(D(\omega, \boldsymbol{k}))+i \operatorname{Im}(D(\omega, \boldsymbol{k}))=0 \\
\operatorname{Re}\left(D\left(\omega_{r}, \boldsymbol{k}\right)\right)+\left.i \Gamma \frac{\partial \operatorname{Re}(D(\omega, \boldsymbol{k}))}{\partial \omega}\right|_{\omega=\omega_{r}} \approx-i \operatorname{Im}\left(D\left(\omega_{r}, \boldsymbol{k}\right)\right) \\
\Gamma \approx-\operatorname{Im}\left(D\left(\omega_{r}, \boldsymbol{k}\right)\right) /\left.\frac{\partial \operatorname{Re}(D(\omega, \boldsymbol{k}))}{\partial \omega}\right|_{\omega=\omega_{r} .} .
\end{gathered}
$$

Separating eq (11) into real and imaginary parts and substituting into eq (16) provides the growth/damping rate:

$$
\Gamma \approx \frac{\psi^{2}}{1+\psi} \sum_{j} \frac{n_{j 0}}{n_{0} \psi_{j} \nu_{j}}\left[\omega_{r}^{2}-\left(\frac{m_{e}}{m_{j}} U_{e}^{2}+\frac{\psi_{j}}{\psi} U_{j}^{2}\right) k^{2}\right] .
$$


The electron drift trigger velocity above which the instability will occur can be found by setting eq (17) to zero, assuming an optimal direction, $\boldsymbol{k} \| \boldsymbol{v}_{\boldsymbol{D}}$, and substituting for the oscillation frequency using (14):

$$
v_{\text {trig }}=(1+\psi) \sqrt{\sum_{j} \frac{n_{j 0}}{\psi_{j} \nu_{j}}\left(\frac{m_{e} \psi_{j}}{m_{j} \psi} U_{e}^{2}+U_{j}^{2}\right) / \sum_{j} \frac{n_{j 0}}{\psi_{j} \nu_{j}}} .
$$

This expression significantly differs from the expression for the 'single species' threshold velocity, $v_{\text {trig }}=(1+\psi) c_{I}$, where $c_{I}$ is the ion-acoustic velocity defined as:

$$
c_{I}^{2} \equiv \sum_{j} \frac{n_{j 0}}{n_{0}}\left(\frac{m_{e}}{m_{j}} U_{e}^{2}+U_{j}^{2}\right) .
$$

In particular, eq (18) replaces the ion-acoustic speed in the single ion trigger velocity equation with a term that couples both thermal and collisional phenomena. From this, we expect the multi-species trigger velocity to be more sensitive to ion-neutral, electron-neutral, and electron-ion collision frequencies than the single species trigger velocity where all of the collisional information is contained within the typically small $\psi$ coefficient.

\subsection{Collision Frequencies}

The trigger velocity of the FBI depends sensitively on the collision rates. In this analysis, we consider three types of elastic collisions: momentum transfer collisions between charged species and neutral hydrogen, resonant charge exchange between protons and neutral hydrogen, and Coulomb collisions between ions and electrons.

First, we consider momentum transfer collisions experienced by all charged species with neutrals. Unfortunately, little experimental data about elastic collisions between neutral hydrogen and metal ion species for energies between $0.1 \mathrm{eV}$ and $1 \mathrm{eV}$ exist. Instead, we must rely upon a relatively simple collision model that can be generalized to any ion species in the solar chromosphere. To do this, we assume the dominant scattering mechanism is the 
repulsive force provided by the dipole polarization of neutral hydrogen when subjected to the electric field produced by the ion species. For charged species $s$ in the neutral frame, Dalgarno et al. (1958) provides this classical result for the momentum transfer collision frequency with a neutral species $n$ :

$$
\nu_{s n}=2.21 \pi n_{n} \sqrt{\frac{\alpha_{n} e^{2}}{4 \pi \epsilon_{0} \mu_{s n}}}
$$

where $n_{n}$ is the number density of the neutral species, $\alpha_{n}$ is the polarizability of the neutral species, $\epsilon_{0}$ is the vacuum permittivity, and $\mu_{s n}$ is the reduced mass of the charged and neutral species. By neglecting quantum effects, this relationship underestimates the collision rates for metal ions.

We can gauge how much eq (20) underestimates the collision frequencies by looking at the relatively few sophisticated calculations performed for some of the lighter metal species at $0.1 \mathrm{eV}$ to $1 \mathrm{eV}$. Kristić and Schultz (2009) and Liu et al. (2010) calculated momentum transfer collision frequencies between Be II and C II with neutral hydrogen using semi-classical and quantum methods. Although Be II is extremely rare in the solar chromosphere, we can still use it to determine the validity of eq (20). Within the energy range of $0.1 \mathrm{eV}$ to $1 \mathrm{eV}$ for Be II and C II, eq (20) underestimates the results of Kristić \& Schultz (2009) and Liu et al. (2010) by $70 \%$ at the greatest. These calculations are more difficult to perform for massive ion species with complicated electronic structures. However, we expect increases in the polarization collision frequencies of heavy ions (e.g. Si II, Mg II, Fe II) to have a minimal effect upon eq (18), the trigger velocity. For example, increasing the collision frequencies for heavy ions by a factor of two only increases our results for eq (18) by $20 \%$ which is not significant enough to factor into the conclusions of this paper. In light of this, we will apply equation (20) to all charged species.

Resonant charge exchange occurs when an ion receives an electron from the neutral with a similar ionization energy, effectively switching the roles of the two. This converts 
fast ions into fast neutrals and vice versa efficiently enough to still be considered elastic collisions for the purpose of this analysis. Resonant charge exchange becomes a significant collision mechanism between protons and neutral hydrogen at temperatures above $300 \mathrm{~K}$ (Banks \& Kockarts 1973). Using a Maxwellian averaged cross-section, Schunk \& Nagy (2009) provides:

$$
\nu_{\text {res }}=2.65 \times 10^{-16} n_{H} \sqrt{\frac{T_{H}+T_{\text {protons }}}{2}}\left[1-0.083 \log _{10}\left(\frac{T_{H}+T_{\text {protons }}}{2}\right)\right]^{2}
$$

where $n_{H}$ has units of $\mathrm{m}^{-3}$.

Coulomb collisions between ions and electrons become important at higher altitudes in the solar chromosphere and can increase the likelihood of the FBI forming there (Gogoberidze et al. 2009). The Coulomb collision frequency for charged species $s$ in the neutral frame, including the Spitzer correction for small angle collisions, is:

$$
\nu_{C o u l, s}=\frac{\pi n_{e} e^{4} \ln \left(12 \pi n_{e} \lambda_{D}\right)}{\left(4 \pi \epsilon_{0}\right)^{2} \sqrt{m_{s}\left(2 K_{B} T\right)^{3}}}
$$

where $\lambda_{D} \approx \sqrt{\epsilon_{0} K_{B} T / n_{e} e^{2}}$ is the Debye length.

The total collision frequency for each charged species, $\nu_{s}$, is the direct sum of eqs (20) and (22). For protons, we add eq (21) to the sum. Figure 2 shows the collision frequencies normalized by the gyrofrequency as a function of pressure within the solar atmosphere.

\section{Results and Discussion}

Combining the multi-species trigger velocity predicted by eq (18) and a semi-empirical, one-dimensional, NLTE model of the solar chromosphere developed by Fontenla et al. (2009) allows one to calculate the trigger velocity for the FBI as a function of pressure. The model provides densities and temperatures for all abundant ion species from hydrogen to zinc as functions of pressure. The dominant ion species just below the temperature 
minimum are Mg II, Si II, and Fe II while protons, and to a lesser extent C II, dominate above as shown in Figure 3. The plasma just below the temperature minimum is unlike any observed in the geospace environment because of the high masses and low mobilities of its dominant ion species. As one moves up from the photosphere, the plasma cools, and the protons and electrons in the plasma begin to recombine leaving a plasma dominated by metals. Before one gets to the temperature minimum, the trend reverses as a result of ionizing radiation from above as demonstrated in Figure 3. Though the Fontenla et al. (2009) model makes detailed predictions, the exact altitude where these changes occur in reality remains uncertain since the model is one-dimensional and has a number of uncertainties.

Figure 4 shows the multi-species trigger velocity as a function of pressure over a range of magnetic field strengths. For this analysis, we adopt a magnetic field range of $30 \mathrm{G}$ to $120 \mathrm{G}$. This range follows from investigations of the Hanle effect in atomic and molecular lines (Trujillo Bueno et al. 2004; 2006). Near the base of the chromosphere, the large neutral density demagnetizes the electrons, producing a large $\psi$ coefficient and preventing the instability from forming. As one moves upward, the trigger velocity rapidly approaches a minimum value near the temperature minimum. The velocity minimum migrates lower into the chromosphere when magnetic field strength increases due to stronger electron magnetization. The multi-species trigger velocity achieves a minimum value of about $7 \mathrm{~km}$ $\mathrm{s}^{-1}$ for $30 \mathrm{G}$ magnetic fields and $4 \mathrm{~km} \mathrm{~s}^{-1}$ for $120 \mathrm{G}$ fields. Note that for the largest estimates of magnetic field strength ( $\gtrsim 75 \mathrm{G}$ ), the model predicts trigger velocities below the neutral acoustic speed. This is significant since one never finds subsonic trigger velocities in the ionosphere, and despite these higher thresholds, the ionospheric FBI is ubiquitous there. Convective overshoots from granular and supergranular flows in the photosphere will drag plasma across magnetic field lines near the temperature minimum. The kinetic energy of these flows should provide enough free energy to induce strong electric fields, producing 
electron drift velocities sufficient to trigger the FBI. This may also provide enough energy to maintain the persistent and global heating contributed by the instability. As one continues upward above the temperature minimum, the trigger velocity increases to approximately 10 $\mathrm{km} \mathrm{s}^{-1}$ for all magnetic field strengths between $30 \mathrm{G}$ and $120 \mathrm{G}$. This increase in trigger velocity makes the instability more difficult to drive, though given the copious amounts of free energy available, it may continue to play a role at high altitudes. A dramatic increase in proton density is responsible for the higher thresholds at these altitudes.

Eq (18) assumes strong electron magnetization and ion demagnetization. Given the substantial range of magnetic fields that exist in the chromosphere, we can check the validity of these assumptions. Figure 2 plots the collision frequency to gyrofrequency ratios for electrons, protons, and Fe II which we use to represent the behavior of all metallic ion species. Near the temperature minimum, electrons appear strongly magnetized while Fe II is strongly demagnetized for all magnetic field strengths. However, the proton frequency ratio ranges from 0.1 to 10 , indicating that protons may potentially magnetize near the temperature minimum. If protons magnetize near the temperature minimum where heavy ions dominate, it may not eliminate the instability, but rather enhance it. Magnetization could limit proton mobility across magnetic field lines, promoting wave growth since the protons would have a more difficult time shorting out the instability in regions where heavy, metallic ions drive the instability. As one moves further up, protons overwhelmingly dominate and become magnetized, shutting off the instability; however, the our predictions from eq (18) do not yet include this physics.

\section{Conclusions}

The multiple ion FBI theory developed above predicts an electron $\boldsymbol{E} \times \boldsymbol{B}$ drift trigger velocity below the neutral acoustic speed for magnetic field strengths greater than $75 \mathrm{G}$. In 
the Earth's auroral electrojet this instability causes intense electron heating and should do the same in the chromosphere. In the chromosphere, the FBI would develop near the solar temperature minimum and extinguish in the upper chromosphere due to the shift from a metal-dominated to a proton-dominated ion population.

Both simulations (Nordlund et al. 1997; Vögler et al. 2005; Freytag et al. 2012) and Hinode observations (Rieutord et al. 2010) show that granule motions at the top of the photosphere drive intense "neutral winds" across solar magnetic field lines. In the Earth's equatorial ionosphere, cross-field neutral winds generate electric fields that easily exceed the Earth's FBI threshold on a daily basis. One would expect that the strong solar flows would create intense electric fields which should easily drive the FBI. As in the Earth's ionosphere, electric fields driven by neutral flows in the solar atmosphere will travel along magnetic field lines; hence, a strong wind across $\boldsymbol{B}$ below the region where the FBI has the lowest threshold may propagate to this region and trigger the instability. Likewise, as in the auroral ionosphere, energy entering the chromosphere from above or below, possibly in the form of Alfvén waves, will manifest itself as strong drifts and electric fields that can trigger the FBI. In the Earth's electrojet, the trigger velocities exceed the both the neutral acoustic and the highest wind speeds but the FBI still occurs because the driving electric field exceeds the local $\boldsymbol{E}=-\boldsymbol{u} \times \boldsymbol{B}$ fields due to a variety of mechanisms. Similarly amplified electric fields may not be necessary to trigger the FBI in the chromosphere, though they may exist.

Directly observing these electric fields will prove difficult, though indirect observations may be possible. In the ionosphere, few in situ measurements of electric fields exist; however, numerous indirect measurements exist (Foster \& Erickson 2000). Near the Earth's electrojets, the field is inferred from ground-based magnetometer measurements since the electric field drives Hall currents which cause easily detected perturbations of 
the geomagnetic field (such changes were among the earliest space-physics measurements, dating to the 1700s). More precise measurements of electric fields are made by radars in the auroral electrojet by detecting field-induced $\boldsymbol{E} \times \boldsymbol{B}$ drifts on field lines above, but connected to, the E-region. At these higher altitudes, electrons and ions drift together due to reduced collision rates. A combination of detailed observations and modeling should allow us to estimate the magnitude of the plasma drifts and fields in the chromosphere.

In this work, we ignored the effects of proton magnetization on the multi-species FBI in the solar chromosphere. Unlike heavier and less mobile ions, protons principally act to short out the perturbed electric fields driving the instability. Magnetizing protons would limit their mobility across magnetic field lines, potentially enhancing the instability. We plan to analyze this more fully in a future work.

This paper further develops the ideas first put forward in Fontenla (2005) and Fontenla et al. (2008) by more accurately modeling the triggering of the FBI in the chromosphere. The argument that this instability should exist in the chromosphere and that it has the potential of being an important mechanism for electron heating in the these regions remains robust. It will operate on rapid time scales much faster than the minute time scales typically considered by MHD systems. Also, its driver will be the neutral flows in the upper photosphere and lower chromosphere, making it a good candidate for heating of the quiet Sun.

This work was supported in part by NSF through Ionospheric Physics Grants Nos. ATM-0442075, ATM-0819914, and ATM-1007789. The authors would like to thank Prof. Javier Trujillo Bueno for bring an error to their attention. 


\section{Figures}

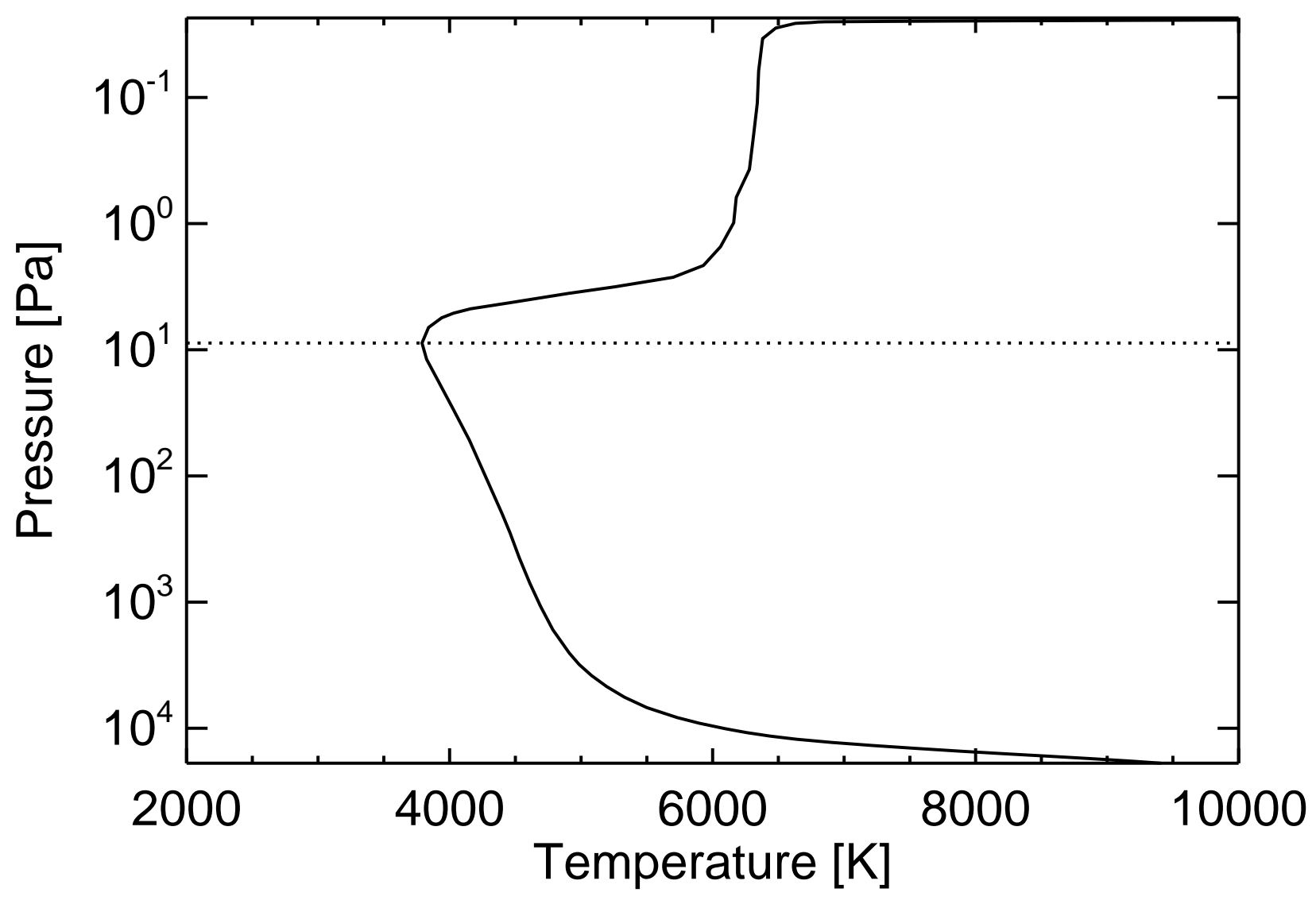

Fig. 1.- Temperature profile of the solar chromosphere from the Fontenla (2009) model. The solar temperature minimum is marked by the dotted line. The temperature increases sharply by over $2000 \mathrm{~K}$ immediately above the temperature minimum. 


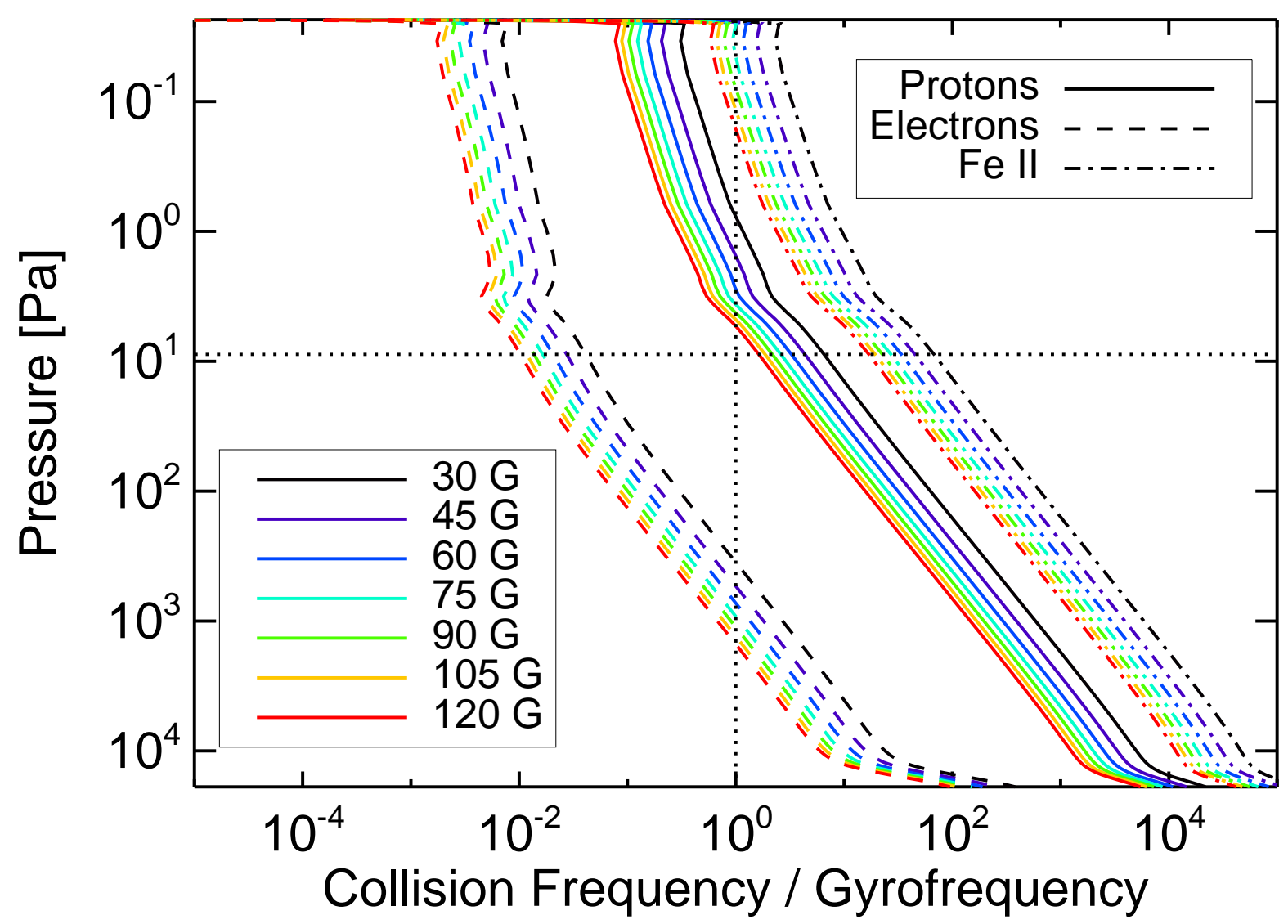

Fig. 2.- Collision frequency normalized to the gyrofrequency for protons, electrons, and Fe II over a range of magnetic field strengths from $30 \mathrm{G}$ to $120 \mathrm{G}$. Values greater than unity imply a demagnetized species. Near the temperature minimum, electrons are strongly magnetized while heavy ions (represented by Fe II) are strongly demagnetized. However, protons may become magnetized near the temperature minimum, potentially enhancing the instability there. 


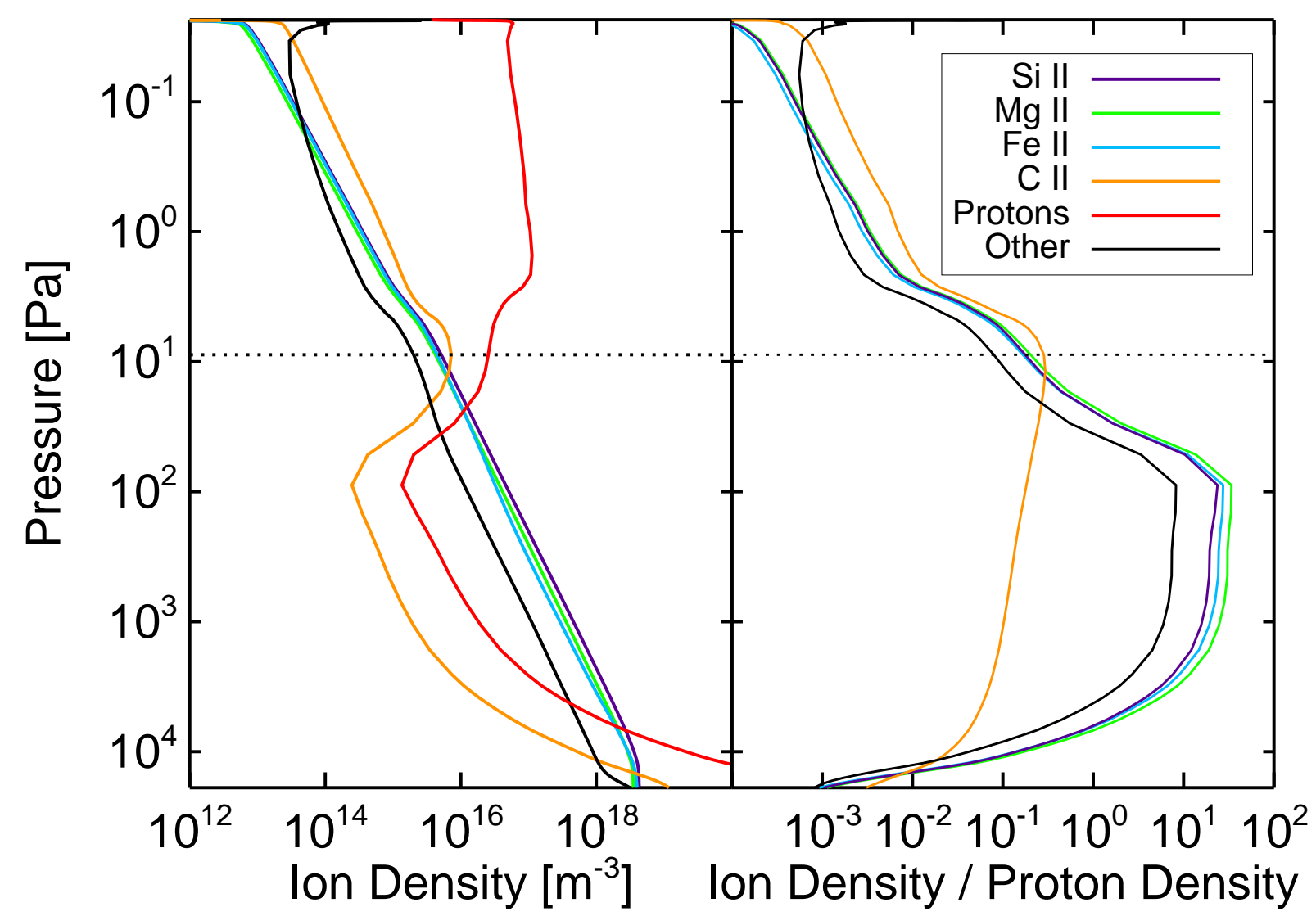

Fig. 3.- Left plot: ion density profiles for Si II (purple), Mg II (green), Fe II (blue), C II (orange), protons (red), and remaining ion species (black) from Fontenla et al. (2009). Right plot: ratios of ion density to proton density for the same ion species. Massive metallic ions (Si II, Mg II, Fe II) dominate below the temperature minimum, while protons, and to a lesser extent C II, dominate above the temperature minimum. 


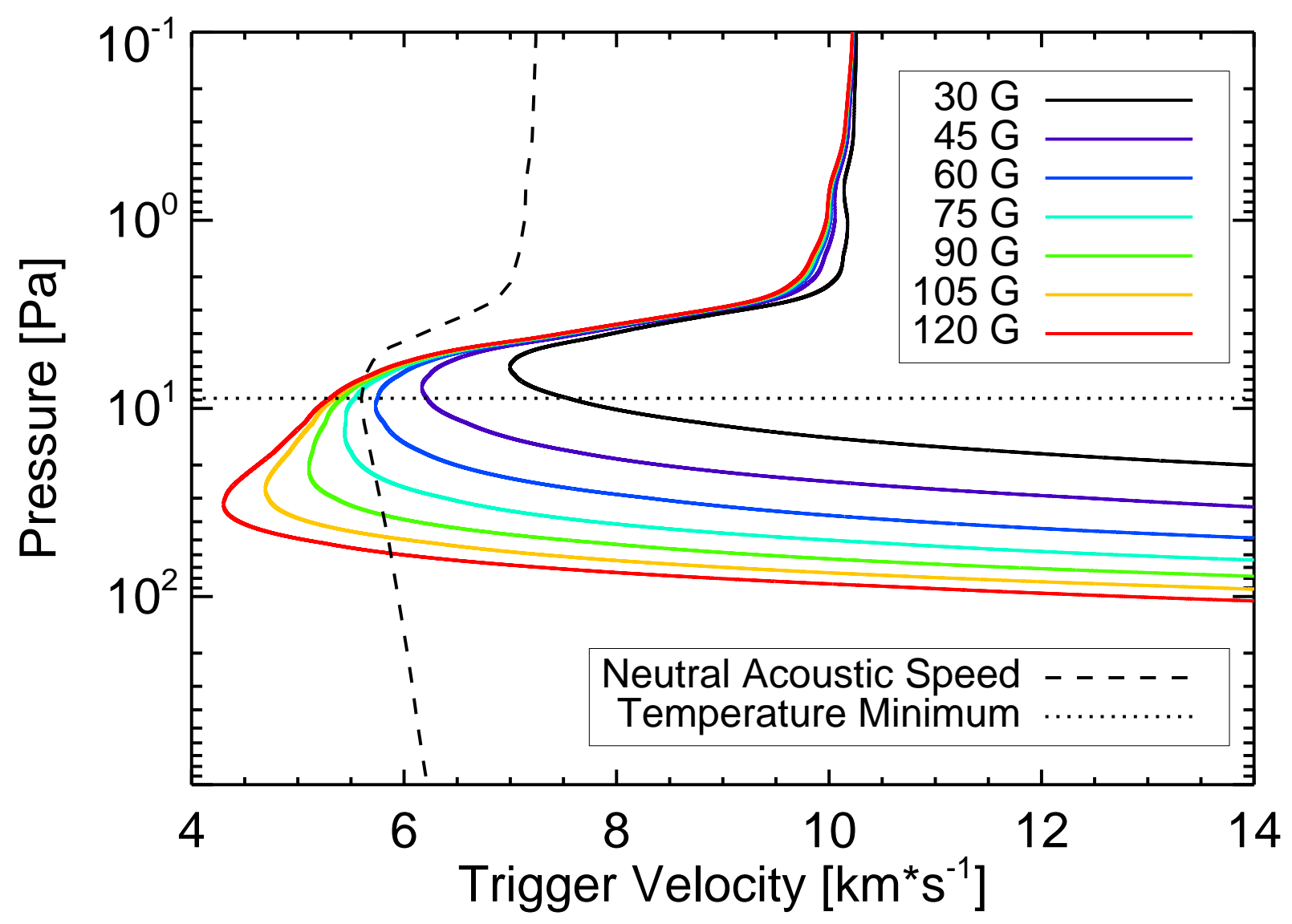

Fig. 4.- Predicted multi-species trigger velocity at several magnetic field strengths. The plot is limited to pressures near the temperature minimum where eq (18) is valid. The trigger velocity decreases sharply as it approaches the temperature minimum reaching speeds as low as $4 \mathrm{~km} \mathrm{~s}^{-1}$ at the largest magnetic field strengths. The neutral acoustic speed is plotted for reference. 


\section{REFERENCES}

Athay, R. G. 1966, ApJ, 146, 223

Banks, P. M., \& Kockarts, G. 1973, Aeronomy, Part A (New York, NY: Academic Press)

Buneman, O. 1963, PhRvL, 10, 285

Campos, L. M. B. C., \& Mendes, P. M. V. M. 1995, MNRAS, 276, 1041

Carlsson, M., \& Stein, R. F. 1992, ApJ, 397L, 59

Carlsson, M. 2007 ASPC, 368, 49

Dalgarno, A., McDowell, M. R. C., \& Williams, A. 1958, RSPTA, 250, 411

Dimant, Y. S., \& Oppenheim, M. M. 2004, JASTP, 66, 1639

Dimant, Y. S., \& Oppenheim, M. M. 2010a, in APS Division of Plasma Physics, 52nd Annual Meeting (Chicago, IL: APS), TO8001D

Dimant, Y. S., \& Oppenheim, M. M. 2010b, in AGU, Fall Meeting (San Francisco, CA: AGU), SM24B-08

Farley, D. T. Jr. 1963, JGR, 68, 6083

Fontenla, J. M., Avrett, E. H., \& Loeser, R. 1991, ApJ, 377, 712

Fontenla, J. M., Avrett, E. H., \& Loeser, R. 1993, ApJ, 406, 319

Fontenla, J. M. 2005, A\&A, 442, 1099F

Fontenla, J. M., Peterson, W. K., \& Harder, J. 2008, A\&A, 480, 839F

Fontenla, J. M., Curdt, W., Haberreiter, M., Harder, J., \& Tian, H. 2009, ApJ, 707, 482 
Foster, J. C., \& Erickson, P. J. 2000, GeoRL, 27, 3177

Freytag, B., Steffen, M., Ludwig, H.-G,. Wedemeyer-Böhm, S., Schaffenberger, W., \& Steiner, O. 2012, CoPh, 231, 919

Gogoberidze, G., Voitenko, Y., Poedts, S., \& Goossens, M. 2009 ApJ, 706L, 12

Kristić, P. S., \& Schultz, D. R. 2009, JPhB, 42, 5207

Liu, C. H., Wang, J. G., \& Janev, R. K. 2010, JPhB, 43, 4006

Nordlund, A., Spruit, H. C., Ludwig, H.-G., \& Trampedach, R. 1997 A\&A, 328, 229

Oppenheim, M. M., \& Dimant, Y. S. 2013, JGRA, 188, 1306

Richmond, A. D. 1973, JATP, 35, 1083

Rieutord, M., Roudier, T., Rincon, F., Malherbe, J.-M., Meunier, N., Berger, T., \& Frank, Z. 2010, A\&A, 512A, 4

Schunk, R., \& Nagy, A. 2009, Ionospheres, (2nd ed.; New York, NY: Cambridge University Press)

Socas-Navarro, H. 2007, ApJS, 169, 439

Sudan, R. N., Akinrimisi, J., \& Farley, D. T. 1973, JGR, 78, 240

Trujillo Bueno, J., Shchukina, N., \& Asensio Ramos, A. 2004, Nature, 430, 326

Trujillo Bueno, J., Asensio Ramos, A., \& Shchukina, N. 2006, ASPC, 358, 269

Vernazza, J. E., Avrett, E. H., \& Loeser, R. 1981, ApJS, 45, 635

Vögler, A., Shelyag, S., Schüssler, M., Cattaneo, F., Emonet, T., \& Linde, T. 2005, A\&A, 429,335 
This manuscript was prepared with the AAS IATEX macros v5.2. 